\title{
Apolipoprotein A-1 Binding Protein Inhibits Inflammatory Signaling Pathways by Binding to Apolipoprotein A-1 in THP-1 Macrophages
}

\author{
Min Zhang, PhD; Guo-Jun Zhao, PhD; Kai Yin, PhD; Xiao-Dan Xia, MD; \\ Duo Gong, MD; Zhen-Wang Zhao, MD; Ling-Yan Chen, MD; \\ Xi-Long Zheng, PhD; Xiao-Er Tang, MD; Chao-Ke Tang, PhD
}

\begin{abstract}
Background: It has previously been demonstrated that apolipoprotein A-1 (apoA-1) binding protein (AIBP) promotes apoA-1 binding to ATP-binding cassette transporter $A 1$ (ABCA1) and prevents ABCA1 protein degradation so as to inhibit foam cell formation. Because apoA-1 inhibits inflammatory signaling pathways, whether AIBP has an inhibitory effect on inflammatory signaling pathways in THP-1-derived macrophages is investigated.
\end{abstract}

Methods and Results: Analysis of inflammation-related gene expression indicated that AIBP decreased lipopolysaccharide (LPS)mediated macrophage inflammation. AIBP significantly prevented NF- $\kappa$ B nuclear translocation. Further, AIBP prevented the activation of mitogen-activated protein kinases (MAPKs), including p38 MAPK, extracellular-signal regulated kinase and c-Jun N-terminal kinase. AIBP decreased MyD88 expression at both mRNA and protein levels, but did not have any effect on TLR4 expression. Moreover, treatment with both AIBP and apoA-1 decreased the abundance of TLR4 in the lipid raft fraction. AIBP lacking 115-123 amino acids ( $\triangle 115-123)$, however, did not have such effects as described for intact AIBP. In addition, knockdown of ABCA1 inhibited the effects of AIBP on inflammatory factor secretion.

Conclusions: These results suggest that AIBP inhibits inflammatory signaling pathways through binding to apoA-1 and stabilizing $A B C A 1$, and subsequent alteration of lipid rafts and TLR4 in the cell membrane.

Key Words: Apolipoprotein A-1 (apoA-1); Apolipoprotein A-1 binding protein (AIBP); ATP-binding cassette transporter A1 (ABCA1); Atherosclerosis; Inflammation

I nflammation plays important roles in the development of atherosclerosis, the main cause of coronary artery disease (CAD). Accumulation of macrophages into the intima correlates with progression of atherosclerotic plaque and plaque rupture. On one hand, macrophages uptake lipids and cholesterol derivatives, resulting in foam cell formation that contributes to fatty streaks. ${ }^{1}$ On the other hand, macrophages are the major contributors to the inflammatory response through their secretion of proinflammatory mediators (chemokines, cytokines, reactive oxygen and nitrogen species) and matrix-degrading proteases and through their eventual cell death, such as necrosis and apoptosis. ${ }^{2}$

Previous studies have suggested that the reverse cholesterol transport (RCT) mediated by high-density lipoprotein (HDL) particles has a relevant anti-atherogenic potential. ${ }^{3}$ In this process, the ATP-binding cassette transporter A1 (ABCA1) releases intracellular cholesterol to apolipoprotein A-1 (apoA-1) to form HDL, which is defined as the first step of RCT. ${ }^{45}$ Our laboratory has also demonstrated that maintaining the abundance of ABCA1 protein can effectively ensure RCT and prevent the development of atherosclerosis. ${ }^{68}$ In addition to its role in cholesterol efflux, $\mathrm{ABCA} 1$ has recently been considered as an anti-inflammation

Received August 16, 2017; revised manuscript received January 9, 2018; accepted February 15, 2018; released online April 3, 2018 Time for primary review: 27 days

Institute of Cardiovascular Disease, Key Lab for Atherosclerology of Hunan Province, Medicine Research Center, Hunan Province Cooperative Innovation Center for Molecular Target New Drug Study, University of South China, Hengyang, Hunan (M.Z., K.Y., X.-D.X., D.G., Z.-W.Z., L.-Y.C., C.-K.T.), China; Department of Histology and Embryology, Guilin Medical University, Guilin, Guangxi (G.-J.Z.), China; Department of Biochemistry and Molecular Biology, The Libin Cardiovascular Institute of Alberta, The University of Calgary, Health Sciences Center, Calgary, Alberta (X.-L.Z.), Canada; Key Laboratory of Molecular Targets \& Clinical Pharmacology, School of Pharmaceutical Sciences, Guangzhou Medical University, Guangzhou, Guangdong (X.-L.Z.), China; and Department of Pathophysiology, Shaoyang University, Shaoyang, Hunan (X.-E.T.), China

The first three authors contributed equally to this work (M.Z., G.-J.Z., K.Y.).

Mailing address: Chao-Ke Tang, PhD, Institute of Cardiovascular Research, University of South China, Hengyang, Hunan 421001, China. E-mail: tangchaoke@qq.com or Xiao-Er Tang, MD, Department of Pathophysiology, Medical College, Shaoyang University, Shaoyang, Hunan 422000, China. E-mail: tangxiaoer19800505@tom.com

ISSN-1346-9843 All rights are reserved to the Japanese Circulation Society. For permissions, please e-mail: cj@j-circ.or.jp 
A

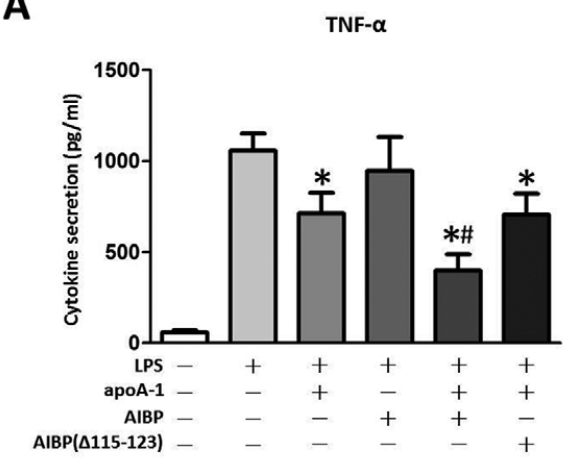

C

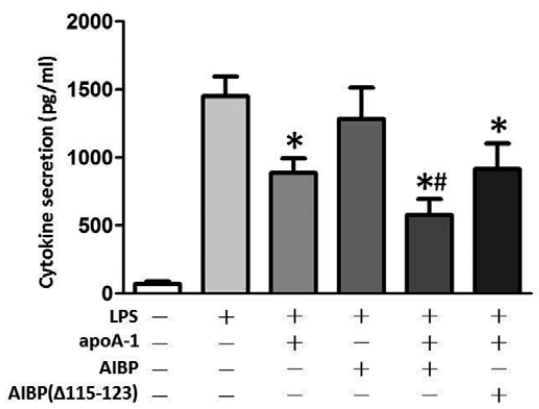

B

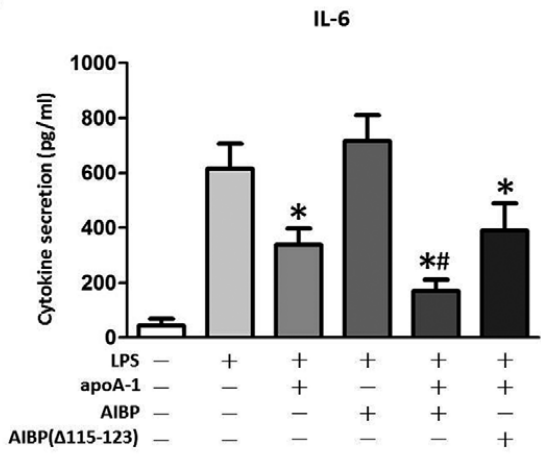

D

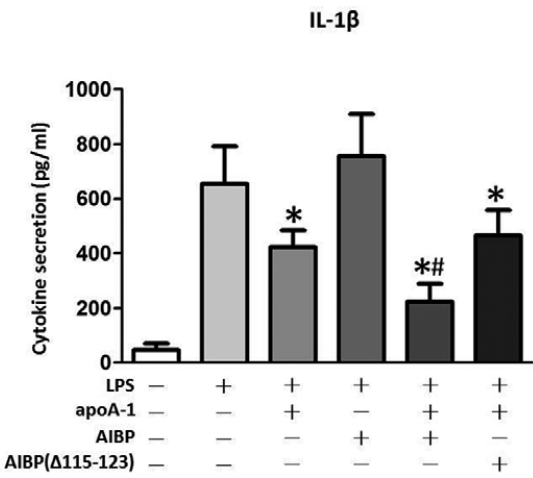

Figure 1. AIBP effects the LPS-stimulated secretion of inflammatory factors in THP-1 macrophages. THP-1 macrophages were incubated with or without $25 \mathrm{mg} / \mathrm{mL}$ apoA-1 and $100 \mathrm{ng} / \mathrm{mL}$ LPS before testing. Secretion levels of TNF- $a$ (A), IL-6 (B), MCP-1 (D) and IL-1 $\beta$ (C) were detected by using an ELISA. Values represent means from 3 individual experiments. * $P<0.05$ vs. the LPS group. \#P<0.05 vs. the apoA-1 group. AIBP, apolipoprotein A-1 binding protein; LPS, lipopolysaccharides; THP-1, a human monocytic cell line derived from an acute monocytic leukemia patient; apoA-1, apolipoprotein A-1; TNF- $a$, tumor necrosis factor- $a$; IL, interleukin; MCP-1, monocyte chemoattractant protein 1; ELISA, enzyme-linked immunosorbent assay.

receptor.69 The patients with dysfunctional ABCA1 and familial HDL deficiencies tend to have chronic inflammation, suggesting that ABCA1 regulation of inflammation is another putative mechanism for its protection against atherosclerosis. ${ }^{\mathbf{1 0}}$

ApoA-1 binding protein (AIBP) is a secreted protein, which was discovered in a screening of the proteins that physically associate with apoA-1.11 We and others have demonstrated that AIBP binding to ABCA1 can enhance macrophage cholesterol efflux in vitro. ${ }^{12,13}$ The $115-123$ amino acids of AIBP were within the Rossmann-like fold and at least partially responsible for its binding to apoA-1 and cholesterol efflux in THP-1-derived macrophages. ${ }^{12}$ Recently, apoA-1 was demonstrated to inhibit inflammation in 3T3-L1 adipocytes by suppressing toll-like receptor 4 (TLR4)-mediated inflammatory signaling pathways. ${ }^{14}$ However, it is not clear whether AIBP has a functional effect on macrophage inflammation. Therefore, this study was designed to investigate the effects of AIBP on macrophage inflammation and explore the possible underlying mechanisms.

\section{Methods}

\section{Cell Culture}

Human THP-1 and HEK 293T cells were obtained from the
Cell Bank of the Chinese Academy of Sciences (Shanghai, China). THP-1 was cultured in RPMI 1640 (Solarbio, China) supplemented with $10 \%$ fetal bovine serum (FBS) at $37^{\circ} \mathrm{C}$ and $5 \% \mathrm{CO}_{2}$. After $3-4$ days, THP-1 cells were treated with phorbol-12-myristate-13-acetate (PMA, $160 \mathrm{nmol} / \mathrm{L}$; Sigma, USA) for $24 \mathrm{~h}$ to differentiate THP-1 to macrophages before they were used in experiments. HEK 293T cells were cultured in Dulbecco's Modified Eagle Medium (DMEM) containing 10\% FBS.

\section{Generation of Recombinant Adeno-Associated Virus}

The gene fragments encoding human AIBP and AIBP $(\Delta 115-$ 123) were generated by reverse transcription polymerase chain reaction (RT-PCR) and overlap extension polymerase chain reaction (OE-PCR) from the $\mathrm{mRNA}$ of human liver cDNA library. Both AIBP and $\operatorname{AIBP}(\Delta 115-123)$ cDNA were cloned into recombinant adeno-associated virus 9 (rAAV9) with a FLAG tag (Cell Biolabs, San Diego, USA) to construct rAAV-AIBP and $\mathrm{rAAV}-\mathrm{AIBP}(\Delta 115-123)$ for amplification. Positive recombinants were loaded into HEK 293 T cells using a rAAV helper-free system (Stratagene, USA) for virus packaging and propagation.

\section{Enzyme-Linked Immunosorbent Assay (ELISA)}

The levels of inflammatory factors were quantitated using an ELISA kit (Boster, China). Briefly, $1 \times 10^{4}$ THP-1 macro- 
A

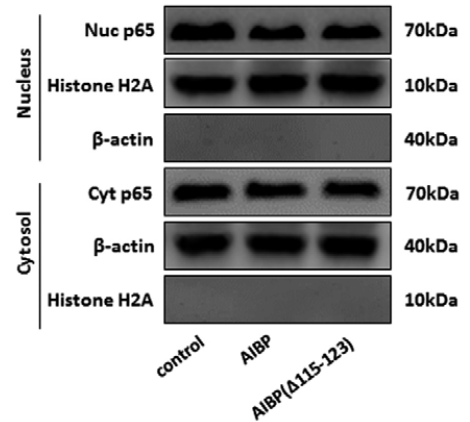

C

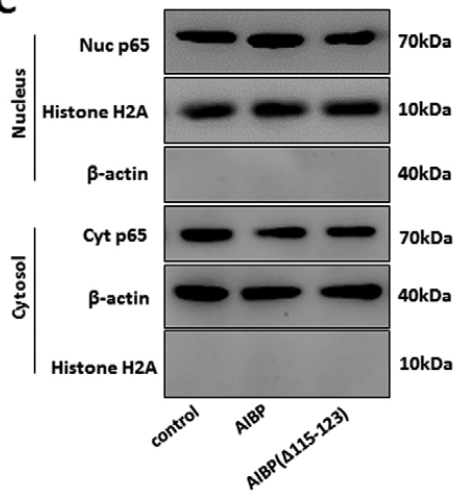

B

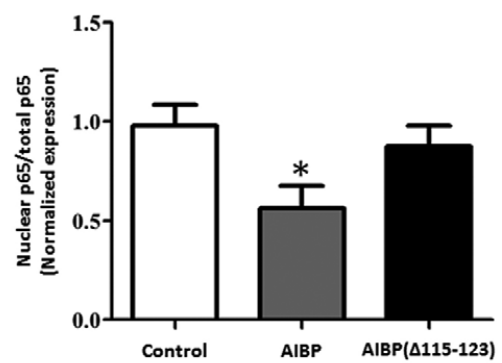

D

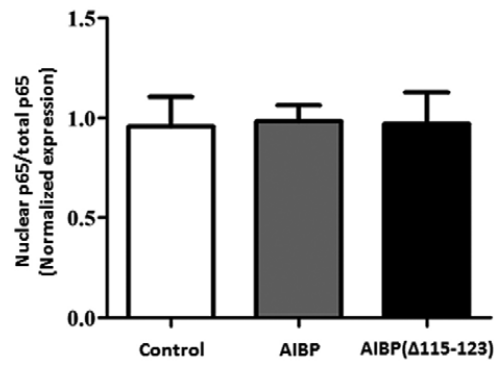

Figure 2. Effects of AIBP on the activation of NF-db. THP-1 macrophages were incubated with $25 \mathrm{mg} / \mathrm{mL}$ apoA-1 and $100 \mathrm{ng} / \mathrm{mL}$ LPS $(\mathbf{A}, \mathbf{B})$ or IL-1 $\beta$ before testing $(\mathbf{C}, \mathbf{D})$. The nuclear and cytoplasmic levels of NF-dB p65 were measured by using Western blotting analysis. ${ }^{*} \mathrm{P}<0.05$ vs. control group. Abbreviations as in Figure 1.

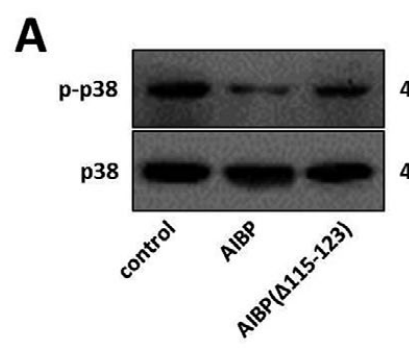

$43 \mathrm{kDa}$

C

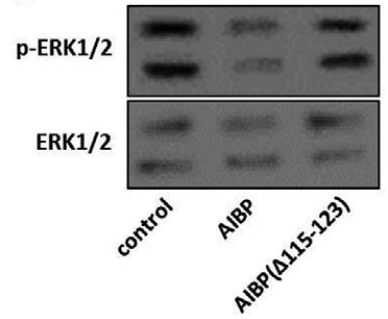

E

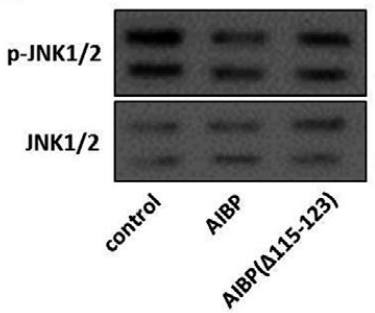

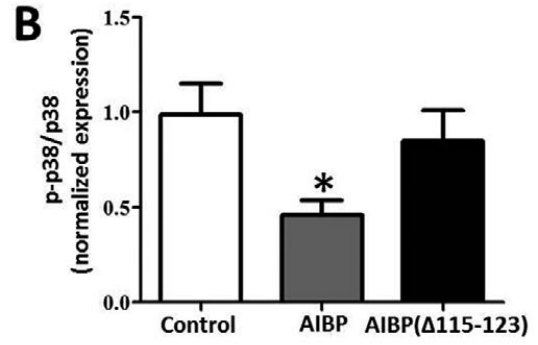

D

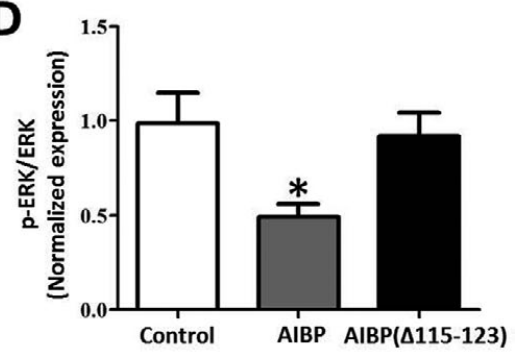

$\mathbf{F}$

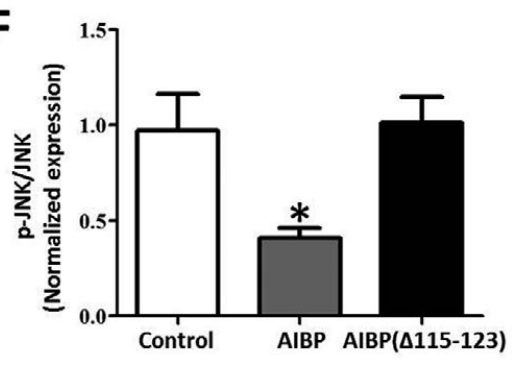

Figure 3. Effects of AIBP on the activation of MAPKs. THP-1 macrophages were incubated with $25 \mu \mathrm{g} / \mathrm{mL}$ apoA-1 and $100 \mathrm{ng} / \mathrm{mL}$ LPS before testing. The levels of p38, p-p38 (A,B), ERK1/2, p-ERK1/2 $(\mathbf{C}, \mathbf{D}), \mathrm{JNK} 1 / 2$ and $p-J N K 1 / 2(\mathbf{E}, \mathbf{F})$ were measured by Western blot analysis. ${ }^{*} \mathrm{P}<0.05$ vs. control group. MAPKs, mitogen-activated protein kinases; ERK, extracellular signal-regulated kinases; JNK, c-Jun N-terminal kinases. Other abbreviations as in Figure 1. 
A

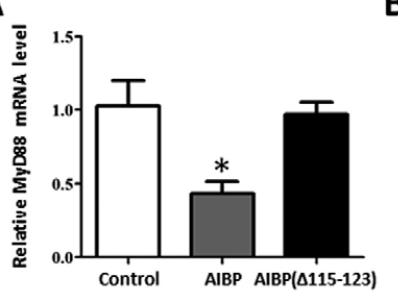

E

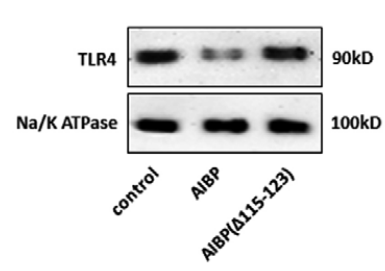

I

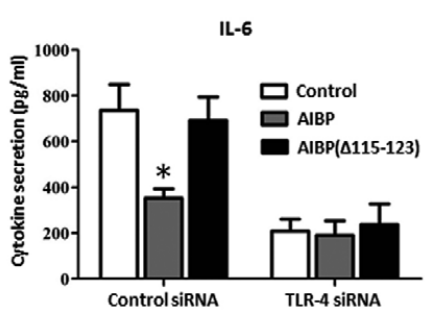

B

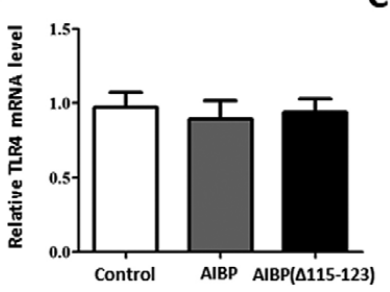

F

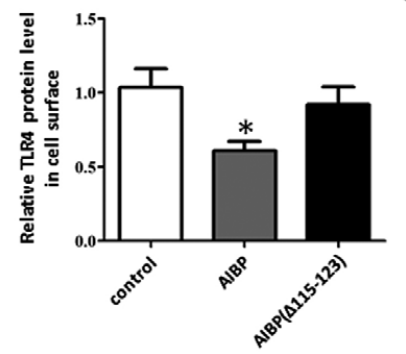

C

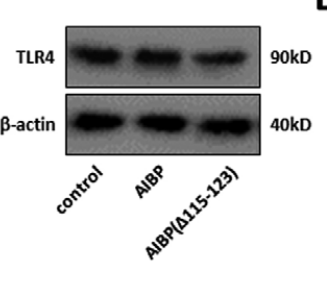

G

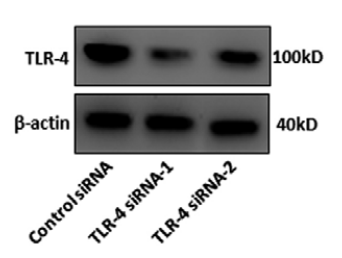

D

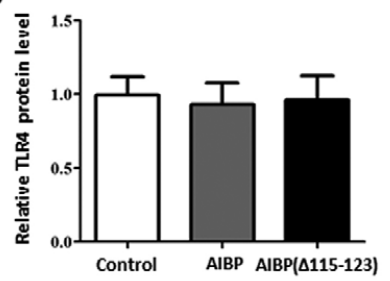

H

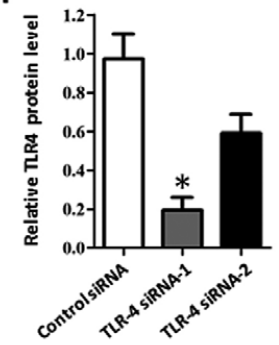

J

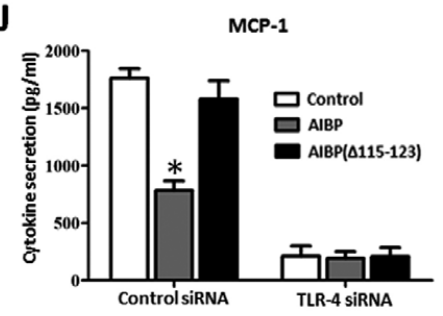

Figure 4. Effects of AIBP on the expression of MyD88 and TLR4. THP-1 macrophages were incubated for $16 \mathrm{~h}$ in the presence of $25 \mu \mathrm{g} / \mathrm{mL}$ apoA-1 and $100 \mathrm{ng} / \mathrm{mL}$ LPS before testing. (A) MyD88 mRNA was detected with qPCR analysis. (B) TLR4 mRNA was detected with qPCR analysis. (C,D) TLR4 proteins were detected by Western blot analysis. (E,F) AlBP effects on TLR4 on the cell surface of the THP-1 macrophage. Following the separation of the cell membrane protein, Western blotting analysis was used to detect TLR4 protein, which was relative to the level of Na/K ATPase in the cell surface. (G,H) TLR4 was silenced with siRNA and its expression was detected by Western blotting analysis. (I,J) After TLR4 was silenced, the effects of AIBP on LPS-induced IL-6 and MCP-1 secretion were examined by using an ELISA. Values are presented as mean \pm SD from 3 individual experiments. ${ }^{*} \mathrm{P}<0.05$ vs. control group. MyD88, myeloid differentiation primary response 88; TLR4, toll-like receptor 4; qPCR, quantitative PCR; ATPase, adenylpyrophosphatase; siRNA, small interfering RNA; ELISA, enzyme-linked immunosorbent assay; SD, standard deviation. Other abbreviations as in Figure 1.

phages were incubated with $100 \mathrm{ng} / \mathrm{mL}$ lipopolysaccharide (LPS) or $10 \mathrm{ng} / \mathrm{mL}$ interleukin- $\beta$ (IL- $1 \beta), 25 \mu \mathrm{g} / \mathrm{mL}$ apoA-1 and $0.2 \mu \mathrm{g} / \mathrm{mL}$ AIBP or $\operatorname{AIBP}(\Delta 115-123)$, followed by the collection of the supernatants. The concentrations of tumor necrosis factor- $\alpha$ (TNF- $\alpha$ ), interleukin-6 (IL-6), monocyte chemoattractant protein-1 (MCP-1), and IL-1 $\beta$ in supernatants were measured by sandwich ELISA following the manufacturer's instructions. The cytokine standards were used to generate standard curves. Quantitative determinations in three different experiments were performed.

\section{Western Blotting Assay}

Protein extracts from tissues or cells were quantified and subjected to sodium dodecyl sulfate-polyacrylamide gel electrophoresis (SDS-PAGE), transferred to polyvinylidene difluoride (PVDF) membranes (Millipore Corporation, USA), and incubated with primary and secondary antibodies, respectively. Primary antibodies were: p65 (Beyotime, 1:500); p38 MAPK (Beyotime, 1:1,000); p-p38 MAPK (Beyotime, 1:1,000); ERK1/2 (Beyotime, 1:1,000); p-ERK1/2 (Beyotime, 1:1,000); JNK1/2 (Beyotime, 1:1,000); p-JNK1/2 (Beyotime,
1:1,000); TLR4 (ABCAM, 1:500); CtBP1 (ABCAM, 1:100); CAV1 (Beyotime, 1:500); ABCA1 (ABCAM, 1:200); Histone H2A (ABCAM, 1:500); and $\beta$-actin, (Proteinteck, 1:2,000). Secondary antibodies were: HRP-labelled Goat Anti-Mouse IgG(H+L) (Beyotime, 1:1,000) and horseradish peroxidase (HRP)-labelled Goat Anti-Rabbit $\operatorname{IgG}(\mathrm{H}+\mathrm{L})$ (Beyotime, 1:1,000). Immunoreactive bands were visualized with Tanon 5500 (China) and BeyoECL Plus (Beyotime, China).

\section{RNA Extraction and Real-Time PCR}

Total RNA in THP-1 macrophages was extracted with the TRIzol reagent and converted to cDNA using PrimeScript RT reagent kits, followed by real-time PCR of MyD88, TLR4 and the housekeeping gene, GAPDH, by TaqMan Gene Expression Assays. The $\Delta \Delta \mathrm{Ct}$ method was used to ascertain differences between genotypes in MyD88 and TLR4 expression level standardized against the reference gene, GAPDH. The sequences of the real-time PCR primers were as follows: human MyD88, 5'-AGGAGGAATCTG TGCTCTACT-3' and 5'-CATTTCATGTCCTGCATGG 
A

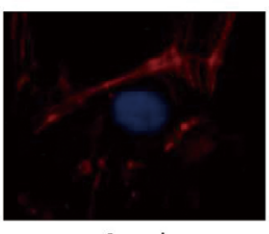

Control

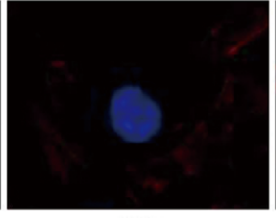

AIBP

C

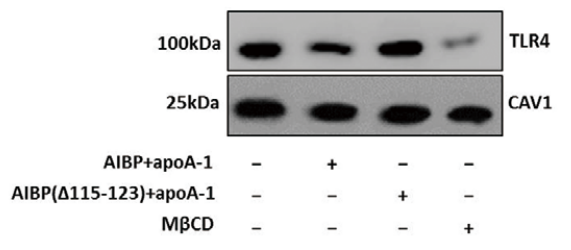

B
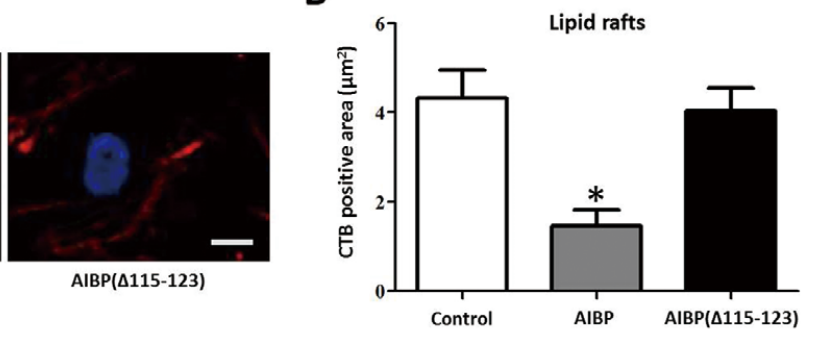

D

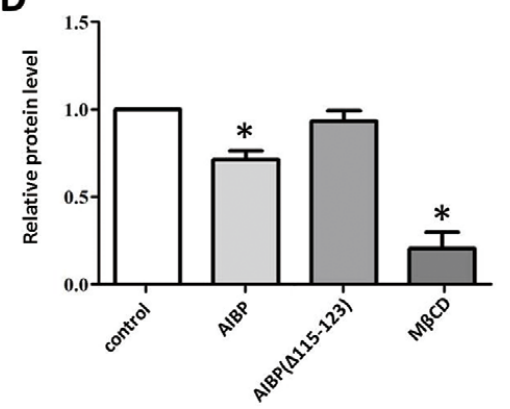

Figure 5. Effects of AIBP on lipid rafts and TLR4 in lipid rafts. (A,B) The effect of AIBP on THP-1 macrophage lipid rafts. THP-1 macrophages were pre-incubated with $25 \mu \mathrm{g} / \mathrm{mL}$ apoA-1, $0.2 \mu \mathrm{g} / \mathrm{mL}$ AIBP or AIBP( $\Delta 115-123)$ for $4 \mathrm{~h}$. The nuclei were stained with DAPI in blue and lipid rafts with cholera toxin B antibody (CTB) and anti-CTB antibody in red. Scale bar, $10 \mathrm{~mm}$. (C,D) TLR4 and CAV1 localization to lipid rafts. THP-1 macrophages were incubated with $20 \mu \mathrm{g} / \mathrm{mL}$ cholesterol-M $\beta C D$ for $6 \mathrm{~h}$, followed by $1-\mathrm{h}$ incubation with or without $25 \mu \mathrm{g} / \mathrm{mL}$ apoA-1 and $0.1 \mu \mathrm{g} / \mathrm{mL}$ AIBP or AIBP $(\Delta 115-123)$ or 30-min incubation with $10 \mathrm{mmol} / \mathrm{L} \mathrm{M} \beta C D$. THP-1 macrophage cell lysates were separated into lipid raft fractions by ultracentrifugation, run on SDS-PAGE and blotted with TLR4 and CAV1 antibodies. *P<0.05 vs. control group. DAPI, 4',6-diamidino-2-phenylindole; CAV1, caveolin-1; M $\beta C D$, methyl- $\beta$ cyclodextrin; SDS-PAGE, sodium dodecyl sulfate-polyacrylamide gel electrophoresis. Other abbreviations as in Figures 1,4.

AACG-3'; human TLR4, 5'-TTTCAGCTCTGCCTTCA CTAC-3' and 5'-GAAAGGTGATTGTTGTGGTGTC-3'; human GAPDH, 5'-CAAGAGCACAAGAGGAAGAG AGA-3' and 5'-CTCCTCACAGTTGCCATGTAG-3'.

\section{Lipid Raft Assay}

Lipid rafts were isolated using a discontinuous gradient ultracentrifugation method with modifications. ${ }^{13}$ Briefly, THP-1 macrophages were washed with ice-cold PBS (placing the container of PBS in ice water). Cells were then scraped from the plate and put into $0.5 \mathrm{~mol} / \mathrm{L}$ sodium carbonate buffer containing a protease inhibitor cocktail before being homogenized and sonicated for $3 \times 10 \mathrm{~s}$. Samples were adjusted to $45 \%$ sucrose by adding a $90 \%$ sucrose solution and then transferred into ultracentrifugation tubes. A 5-30\% sucrose discontinuous gradient was formed above the sample carefully, followed by ultracentrifugation at $20,000 \mathrm{~g}$ for $18 \mathrm{~h}$ at $4^{\circ} \mathrm{C}$ in a MAX-130K (Beckman Optima, USA). Ten fractions were collected from the top to the bottom. The lipid rafts fraction (fraction 5) was used for SDS-PAGE with TLR4 antibody (ABCAM, 1:500) or CAV1 antibody (Beyotime, 1:500).

\section{Visualization of Lipid Rafts}

The visualization of lipid rafts was performed as previously described, but with modifications. ${ }^{13}$ THP-1 macrophages were plated on glass coverslips and pre-incubated with $25 \mu \mathrm{g} / \mathrm{mL}$ apoA-1, $0.2 \mu \mathrm{g} / \mathrm{mL}$ AIBP or $\operatorname{AIBP}(\Delta 115-123)$ for $24 \mathrm{~h}$. Cells were washed once with medium. Then $1 \mu \mathrm{g} / \mathrm{mL}$
Alexa Fluor 594-labeled cholera toxin B (CTB, Invitrogen) for $15 \mathrm{~min}$ at $4^{\circ} \mathrm{C}$ was added to the cells. The cells were washed with PBS and incubated with the anti-CTB antibody (ABCAM, USA, 1:100) for $15 \mathrm{~min}$ at $4^{\circ} \mathrm{C}$ to crosslink CTB and the lipid raft. After washing with PBS, cells were fixed with $4 \%$ formaldehyde for $15 \mathrm{~min}$. Nuclear DNA was labeled with a 4',6-diamidino-2-phenylindole (DAPI) staining solution (Beyotime, China) and photographed with an Olympus IX73 fluorescent microscope.

\section{Transfection of siRNA}

Pre-designed siRNA-targeting ABCA1 and the corresponding control siRNAs were purchased from Biology Engineering Corporation (Shanghai, China). THP-1 macrophages $\left(2 \times 10^{6}\right.$ cells/well $)$ were transfected with the siRNA of ABCA1 or control siRNA using Lipofectamine 2000 (Invitrogen) according to the manufacturer's protocol. After $48 \mathrm{~h}$ incubation, ABCA1 protein level was detected using Western blot analysis.

\section{Statistical Analysis}

All data are presented as means \pm SD and evaluated using Student's t-test of 2 groups ( 2 tailed) or one-way ANOVA with Tukey's post-hoc test or Dunnett's post-hoc test. A value of $\mathrm{P}<0.05$ was considered statistically significant. All the statistical analyses were performed using Prism software (GraphPad Software, La Jolla, CA). 
A

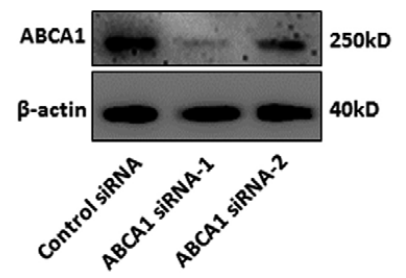

D

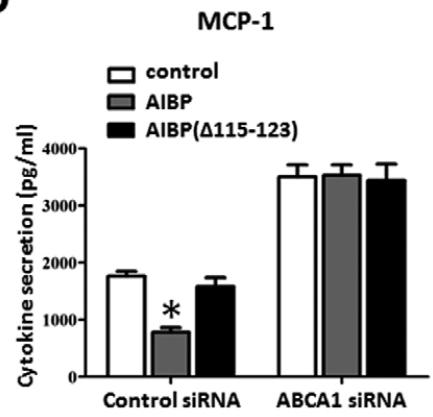

B

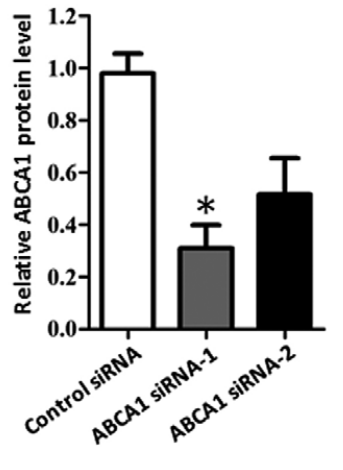

E

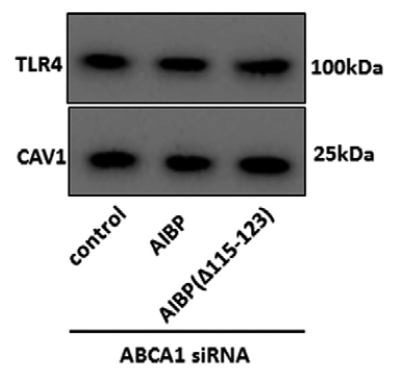

C IL-6

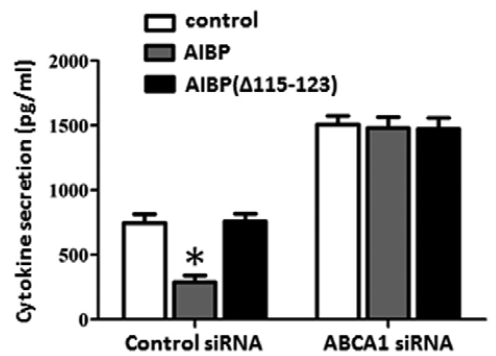

F

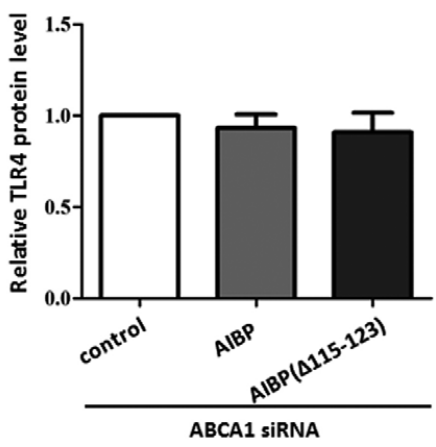

Figure 6. ATP-binding cassette transporter $A 1$ (ABCA1) is involved in the downregulation of inflammatory factor expression induced by AIBP. (A,B) The silencing effect of ABCA1 siRNA. Western blotting approach was used to detect the silencing efficiency of ABCA1. (C,D) The effects of AIBP on LPS-stimulated inflammatory factor secretion from THP-1 cells. Both IL-6 and MCP-1 secretion levels were detected by using an ELISA. (E,F) The effects of AIBP on TLR-4 localization in lipid rafts after ABCA1 was silenced. Both TLR-4 and CAV1 levels in lipid rafts were measured by Western blot analysis. Values represent mean \pm SD from 3 individual experiments. ${ }^{*}>0.05$ vs. control group. Abbreviations as in Figures $1,4,5$.

\section{Results}

\section{AIBP Inhibits the Secretion of Proinflammatory Cytokines Induced by LPS}

Macrophage inflammatory response is a key component in the development of atherosclerosis. To investigate whether AIBP has an effect on macrophage inflammation, we detected the secretion of inflammatory factors in THP-1 macrophages induced by LPS after AIBP treatment. Our results revealed that apoA-1 prevented LPS-stimulated expression and secretion of proinflammatory factors, such as tumor necrosis factor- $\alpha$ (TNF- $\alpha$ ), interleukin 6 (IL-6), monocyte chemotactic protein 1 (MCP1) and interleukin-1 $\beta$ (IL-1 $\beta$ ). Co-treatment with AIBP and apoA-1 further inhibited the secretion of inflammatory factors, suggesting AIBP has significant anti-inflammatory functions (Figure 1). However, AIBP lacking amino acids from 115 to 123 $(\Delta 115-123)$ did not have any effect on inflammatory factor secretion (Figure 1). We previously showed that this domain was responsible for AIBP binding to apoA-1. ${ }^{2}$ Importantly, similar results were also obtained with HepG2 cells (Figure S1). Taken together, our results demonstrated that AIBP inhibits the secretion of proinflammatory cytokines induced by LPS through binding to apoA-1.

AIBP Inhibits Nuclear Translocation of NF- $к B$

$\mathrm{NF}-\kappa \mathrm{B}$ is a key regulator of proinflammatory factor expression in macrophages. We then detected the effect of AIBP on nuclear translocation of NF- $\kappa$ B after treatment of THP-1 cells with LPS. As shown in Figure 2A and B, AIBP could significantly prevent NF- $\kappa$ B nuclear translocation. However, AIBP did not effect the IL- $1 \beta$-induced NF- $\kappa \mathrm{B}$ nuclear translocation (Figure 2C,D). Taken together, these results suggest that AIBP might prevent LPS-induced macrophage inflammation by inactivating the NF- $\kappa \mathrm{B}$ pathway.

\section{AIBP Inhibits the Activation of Mitogen-Activated Protein Kinases (MAPKs)}

MAPKs are important upstream regulators of the NF- $\kappa \mathrm{B}$ signaling pathway in inflammatory and immune responses. ${ }^{15,16}$ As shown in Figure 3, AIBP could prevent the activation of MAPKs including p38 MAPK (p38), extracellular-signal regulated kinase (ERK) and c-Jun $\mathrm{N}$-terminal kinase (JNK). The results suggest that AIBP inactivates NF- $\kappa$ B and reduces inflammation likely through suppressing the MAPK activation.

\section{Effect of AIBP on MyD88 and TLR4 Expression}

TLR4, a known activator of MAPKs, plays an important role in innate immune signaling. ${ }^{14}$ Activation of TLR4, the main receptor for bacterial LPS, is associated with lipidinduced inflammation. ${ }^{17,18}$ As the major adaptor protein of TLR4, myeloid differentiation factor 88 (MyD88) 


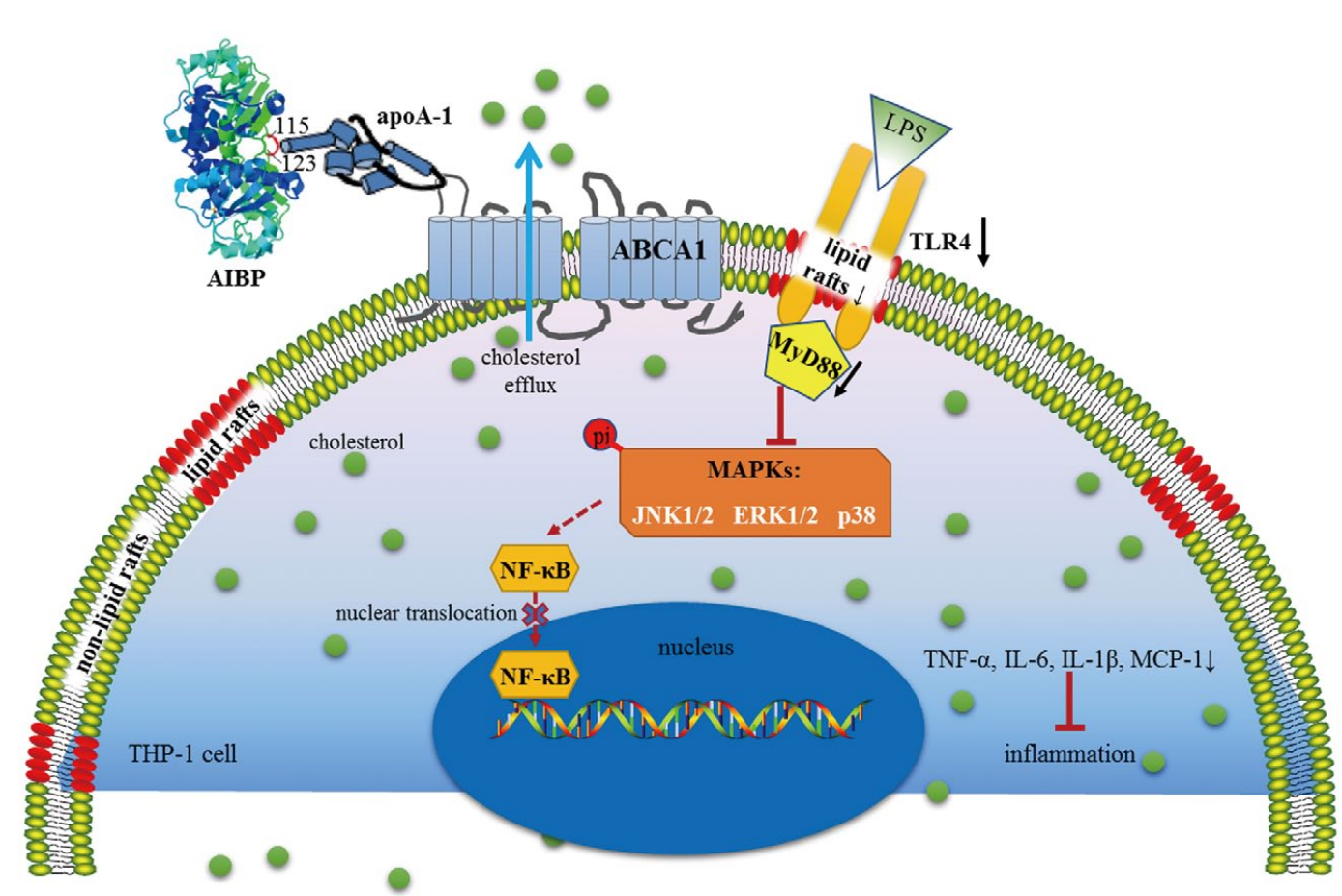

Figure 7. Schematic illustration of the effects of AIBP on ABCA1-dependent cholesterol efflux and TLR-4/NF-kB pathway-mediated inflammation. The present study has unraveled the following mechanisms: AIBP inhibits inflammatory signaling pathways through binding to apoA-1 and stabilizing ABCA1, and subsequent alteration of lipid rafts and TLR4 in the cell membrane, the decreasing TLR4 then inhabited the MAPKs phosphorylation and NF-kB nuclear translocation. Abbreviations as in Figures 1-4,6.

activates MAPKs and NF- $\kappa \mathrm{B}$ to induce inflammation. ${ }^{19}$ Our results showed that AIBP decreased MyD88 expression (Figure 4A). However, AIBP did not have any effect on TLR4 expression at both mRNA and protein levels (Figure 4B-D). Further study revealed that AIBP reduced the TLR4 level on the cell surface (Figure 4E,F), suggesting that AIBP-induced cholesterol efflux alters the abundance of TLR4 in the cell membrane. In addition, we used TLR4 siRNA to knock down its protein expression (Figure 4G,H), and then tested the expression of inflammatory factors in the presence of LPS (Figure 4I, J). The results showed that AIBP did not significantly effect LPS-induced secretion of inflammatory factors when TLR4 was downregulated, suggesting the anti-inflammatory action of AIBP was mainly dependent upon TLR4.

ABCA1-mediated cholesterol efflux from the cell membrane, which disrupts the plasma membrane lipid raft (cholesterol-rich membrane microdomains), has been found to affect signaling on the membrane receptor. ${ }^{\mathbf{1 9 , 2 0}}$ We have demonstrated that AIBP could increase the ABCA1 expression and the cholesterol efflux via binding to apoA-1.12 We then determined whether AIBP could affect lipid raft in the plasma membrane. Cholera toxin B-marked lipid rafts were significantly reduced after AIBP treatment (Figure 5A,B). Lipid rafts were then extracted from THP-1-derived macrophage cell membranes by discontinuous density gradient ultracentrifugation. We observed that AIBP plus apoA-1 treatment, similar to methyl- $\beta$-cyclodextrin ( $\mathrm{M} \beta \mathrm{CD})$, decreased the abundance of TLR4 in the lipid raft fraction (Figure 5C,D). However, $\operatorname{AIBP}(\Delta 115-123)$ did not affect the distribution of TLR4 on lipid rafts. These results suggest that AIBP decreases inflammation through the TLR4-MyD88-dependent pathway.

\section{AIBP Decreases Inflammation in an ABCA1-Dependent Manner}

To determine the role of ABCA1 in AIBP-mediated inhibition of inflammation, we knocked-down the expression of ABCA1 in THP-1 macrophages (Figure 6A,B). After ABCA1 was silenced, AIBP did not lower inflammatory factor secretion (Figure 6C,D) and affect the localization of TLR4 in lipid rafts (Figure 6E,F), suggesting that AIBP alters lipid rafts and affects TLR4 in the cell membrane to decrease inflammation through the mechanism involving ABCA1.

\section{Discussion}

Atherosclerosis and the subsequent pathological changes are caused by lipid metabolism disorder and chronic inflammation of blood vessels, and is an important cause of CAD, myocardial infarction, stroke, peripheral arterial disease and other vascular events. ${ }^{21,22}$ Innate immunity plays an important role in the development of atherosclerosis. Monocytes/macrophages are the main immune cells of the innate immune response and have the promoting effect on the development of atherosclerosis. ${ }^{23}$ In this study, we reported that AIBP inhibits macrophage innate immunity by binding to apoA-1 (Figure 7), suggesting its potential role in prevention and treatment of atherosclerosis.

ApoA-I exerts anti-inflammatory effects by transporting cholesterol out from the cells and binding it with the 
ABCA1. ${ }^{14,24}$ AIBP could promote apoA-1 binding to ABCA1 in macrophages. ${ }^{12}$ Thus, we speculated that the combination of AIBP and apoA-1 may modulate inflammatory responses by influencing inflammatory signaling pathways. TLR4, an important receptor protein of LPS, plays an important role in mediating inflammatory signaling pathways and influences the development of cardiovascular diseases. ${ }^{25}$ We showed that AIBP did not have an effect on TLR4 expression at both mRNA and protein levels, but decreased the abundance of TLR4 in the cell membrane. These results were consistent with the effect of apoA-1 on TLR4 in 3T3-L1 cells. ${ }^{14}$ Recruitment of TLR4 into lipid rafts is critical for the inflammatory response induced by LPS. ${ }^{26}$ In this study, AIBP could decrease the content of lipid rafts marked by CTB and increase the recruitment of TLR4 into lipid rafts. To sum up, AIBP alters the lipid raft content of macrophages by modulating ABCA1-mediated cholesterol efflux, reducing the amount of TLR4 into cell membrane lipid rafts, and then inhibiting inflammatory responses.

MyD88, an adaptor of TLR4, can activate signaling pathways downstream of members of the TLR and IL-1 receptor families, leading to activation of the pathways including NF- $\kappa \mathrm{B}$ and MAPKs., ${ }^{9,27} \mathrm{NF}-\kappa \mathrm{B}$ is an important transcription factor that regulates the expression of inflammatory factors and plays important roles in various stages of atherosclerosis. ${ }^{28}$ In response to an inflammatory stimulus, such as LPS, the NF- $\kappa$ B p65 subunits translocate to the nucleus where they increase transcription of inflammatory genes, such as MCP-1 and IL-6. ${ }^{29,30}$ In the present study, we showed that AIBP can inhibit activation of NF- $\kappa$ B and the secretion of TNF- $\alpha$, IL- 6, MCP1, and IL- $1 \beta$, suggesting an important role for NF- $\kappa$ B in AIBP inhibition of inflammation. MAPK signaling cascades likely play an important role in the pathogenesis of atherosclerosis. ${ }^{31}$ Our research revealed that AIBP can significantly inhibit the LPSmediated phosphorylation of ERK, JNK, and p38. Taken together, AIBP inhibits the LPS-induced inflammatory response of macrophages via inactivation of NF- $\kappa \mathrm{B}$ and MAPK signaling pathways.

ABCA1 may be involved in inflammatory regulation through several inflammatory signaling pathways. ${ }^{32,33}$ The interaction of apoA-1 with ABCA1 expressed in macrophages suppressed the ability of LPS to induce the expression of the inflammatory cytokines, which was reversed by silencing STAT3 or ABCA1. 33,34 We previously demonstrated that apoA-1 suppresses CD40 proinflammatory signaling by preventing TRAF6 translocation to lipid rafts through ABCA1-dependent regulation of free cholesterol efflux. ${ }^{35}$ Downregulation of ABCA1 by siRNA abolished the activation of endothelial nitric oxide synthase (eNOS) and heme oxygenase-1 (HO-1) triggered by the apoA-1 mimetic peptide, D-4F, in endothelial cells. ${ }^{36}$ However, whether AIBP regulates the inflammatory response mediated by STAT3, CD40, eNOS, HO-1 or other signaling pathways remains to be further investigated.

We demonstrated that the effects of AIBP on TLR4, MyD88, NF- $\kappa \mathrm{B}$ and MAPK signaling pathways were apoA-1- and ABCA1-dependent in human monocytederived macrophages. The effects were consistent with the findings by $\mathrm{Wu}$ et a ${ }^{37}$ who found that apoA-1 decreases TLR2 and NF- $\kappa$ B activation in an ABCA1-dependent manner in macrophages. In 3T3-L1 adipocytes, however, the inhibitory effects of apoA-1 on the LPS-induced nuclear localization of the NF- $\kappa \mathrm{B}$ p65 subunits and MAPK signaling pathways were independent of ABCA1. ${ }^{14}$ The reason for this discrepancy remains unknown and may be related to different cell lines.

AIBP has a Rossmann-like fold located in a YjeF_N domain, which is a crucial domain for the protein family involved in cholesterol processing and steroid hormone metabolism. Previously, we demonstrated that AIBP 115123 amino acids, localized in the Rossmann-like fold, are at least partially responsible for its binding to apoA-1. ${ }^{12} \mathrm{In}$ this study, we found that AIBP could not suppress the secretion of inflammatory factors in the $\operatorname{AIBP}(\Delta 115-123)$ group. Furthermore, the effect of AIBP on NF- $\kappa$ B and MAPK signaling pathways was also abolished when amino acids from 115 to 123 were absent. These results showed that AIBP inhibits inflammatory signaling pathways by binding to apoA-1 in THP-1 macrophages.

In conclusion, this report provides new insights into the mechanisms for AIBP to suppress the inflammatory factor secretion in macrophages. Given that AIBP inhibits cholesterol efflux and foam cell formation, it is possible that AIBP may be a potential novel promising therapeutic strategy to inhibit inflammation and lipid accumulation in macrophages, thereby decreasing the development of atherosclerosis.

\section{Acknowledgments}

The authors gratefully acknowledge the financial support from the National Natural Sciences Foundation of China (81570408, 81770461), and the University of South China Innovation Foundation for Postgraduates (2016XCX04), Natural Science Foundation of Hunan Province (2018JJ3451).

\section{References}

1. Yu XH, Fu YC, Zhang DW, Yin K, Tang CK. Foam cells in atherosclerosis. Clin Chim Acta 2013; 424: 245-252.

2. Moore KJ, Sheedy FJ, Fisher EA. Macrophages in atherosclerosis: A dynamic balance. Nat Rev Immunol 2013; 13: 709-721.

3. Huang L, Fan B, Ma A, Shaul PW, Zhu H. Inhibition of ABCA protein degradation promotes HDL cholesterol efflux capacity and RCT and reduces atherosclerosis in mice. J Lipid Res 2015; 56: $986-997$.

4. Ogura M, Ayaori M, Terao Y, Hisada T, Iizuka M, Takiguchi $\mathrm{S}$, et al. Proteasomal inhibition promotes ATP-binding cassette transporter $\mathrm{A} 1$ (ABCA1) and ABCG1 expression and cholesterol efflux from macrophages in vitro and in vivo. Arterioscler Thromb Vasc Biol 2011; 31: 1980-1987.

5. Zhao GJ, Yin K, Fu YC, Tang CK. The interaction of ApoA-I and ABCA1 triggers signal transduction pathways to mediate efflux of cellular lipids. Mol Med 2012; 18: 149-158.

6. Yin K, Tang SL, Yu XH, Tu GH, He RF, Li JF, et al. Apolipoprotein A-I inhibits LPS-induced atherosclerosis in ApoE(-l-) mice possibly via activated STAT3-mediated upregulation of tristetraprolin. Acta Pharmacol Sin 2013; 34: 837-846.

7. Lv YC, Tang Y, Zhang P, Wan W, Yao F, He P, et al. Histone methyltransferase enhancer of zeste homolog 2-mediated ABCA1 promoter DNA methylation contributes to the progression of atherosclerosis. PLoS One 2016; 11: e157265.

8. Yin K, You Y, Swier V, Tang L, Radwan MM, Pandya AN, et al. Vitamin D protects against atherosclerosis via regulation of cholesterol efflux and macrophage polarization in hypercholesterolemic swine. Arterioscler Thromb Vasc Biol 2015; 35: 2432 2442.

9. Ito A, Hong C, Rong X, Zhu X, Tarling EJ, Hedde PN, et al. LXRs link metabolism to inflammation through Abca1-dependent regulation of membrane composition and TLR signaling. Elife 2015; 4: e8009.

10. Soro-Paavonen A, Westerbacka J, Ehnholm C, Taskinen MR Metabolic syndrome aggravates the increased endothelial activation and low-grade inflammation in subjects with familial low HDL. Ann Med 2006; 38: 229-238.

11. Ritter M, Buechler C, Boettcher A, Barlage S, Schmitz-Madry A, Orso E, et al. Cloning and characterization of a novel apoli- 
poprotein A-I binding protein, AI-BP, secreted by cells of the kidney proximal tubules in response to HDL or ApoA-I. Genomics 2002; 79: 693-702

12. Zhang M, Li L, Xie W, Wu JF, Yao F, Tan YL, et al. Apolipoprotein A-1 binding protein promotes macrophage cholesterol efflux by facilitating apolipoprotein A-1 binding to ABCA1 and preventing ABCA1 degradation. Atherosclerosis 2016; 248: 149_ 159.

13. Fang L, Choi SH, Baek JS, Liu C, Almazan F, Ulrich F, et al. Control of angiogenesis by AIBP-mediated cholesterol efflux. Nature 2013; 498: 118-122.

14. Sultana A, Cochran BJ, Tabet F, Patel M, Torres LC, Barter PJ et al. Inhibition of inflammatory signaling pathways in 3T3-L1 adipocytes by apolipoprotein A-I. FASEB $J$ 2016; 30: 2324 2335 .

15. Kim KN, Ko SC, Ye BR, Kim MS, Kim J, Ko EY, et al. 5-Bromo-2-hydroxy-4-methyl- benzaldehyde inhibited LPSinduced production of pro-inflammatory mediators through the inactivation of ERK, p38, and NF-kappaB pathways in RAW 264.7 macrophages. Chem Biol Interact 2016; 258: 108-114.

16. Zhang X, Li N, Shao H, Meng Y, Wang L, Wu Q, et al. Methane limit LPS-induced NF-kappaB/MAPKs signal in macrophages and suppress immune response in mice by enhancing PI3K/AKT/ GSK-3 $\beta$-mediated IL-10 expression. Sci Rep 2016; 6: 29359.

17. Abate W, Alghaithy AA, Parton J, Jones KP, Jackson SK. Surfactant lipids regulate LPS-induced interleukin-8 production in A549 lung epithelial cells by inhibiting translocation of TLR4 into lipid raft domains. J Lipid Res 2010; 51: 334-344.

18. Schoeniger A, Fuhrmann H, Schumann J. LPS- or Pseudomonas aeruginosa-mediated activation of the macrophage TLR4 signaling cascade depends on membrane lipid composition. PeerJ 2016; 4: e1663.

19. Jang SE, Hyam SR, Jeong JJ, Han MJ, Kim DH. Penta-Ogalloyl-beta-D-glucose ameliorates inflammation by inhibiting MyD88/NF-kappaB and MyD88/MAPK signalling pathways. Br J Pharmacol 2013; 170: 1078-1091.

20. Fessler MB, Parks JS. Intracellular lipid flux and membrane microdomains as organizing principles in inflammatory cell signaling. J Immunol 2011; 187: 1529-1535.

21. Taleb S. Inflammation in atherosclerosis. Arch Cardiovasc Dis 2016; 109: 708-715.

22. Viola J, Soehnlein O. Atherosclerosis: A matter of unresolved inflammation. Semin Immunol 2015; 27: 184-193.

23. Mendel I, Yacov N, Harats D, Breitbart E. Therapies targeting innate immunity for fighting inflammation in atherosclerosis. Curr Pharm Des 2015; 21: 1185-1195.

24. Murphy AJ, Woollard KJ, Hoang A, Mukhamedova N, Stirzaker RA, McCormick SP, et al. High-density lipoprotein reduces the human monocyte inflammatory response. Arterioscler Thromb Vasc Biol 2008; 28: 2071-2077.

25. Moghimpour BF, Vallejo JG, Rezaei N. Toll-like receptor signaling pathways in cardiovascular diseases: Challenges and opportunities. Int Rev Immunol 2012; 31: 379-395.

26. Zhang Y, Chen F, Chen J, Huang S, Chen J, Huang J, et al.
Soyasaponin $\mathrm{Bb}$ inhibits the recruitment of toll-like receptor 4 (TLR4) into lipid rafts and its signaling pathway by suppressing the nicotinamide adenine dinucleotide phosphate (NADPH) oxidase-dependent generation of reactive oxygen species. $\mathrm{Mol}$ Nutr Food Res 2016; 60: 1532-1543.

27. Das S, Alhasson F, Dattaroy D, Pourhoseini S, Seth RK, Nagarkatti M, et al. NADPH oxidase-derived peroxynitrite drives inflammation in mice and human nonalcoholic steatohepatitis via TLR4-lipid raft recruitment. Am J Pathol 2015; 185: 1944-1957.

28. Pamukcu B, Lip GY, Shantsila E. The nuclear factor--kappa B pathway in atherosclerosis: A potential therapeutic target for atherothrombotic vascular disease. Thromb Res 2011; 128: $117-$ 123.

29. Li Q, Verma IM. NF-kappaB regulation in the immune system. Nat Rev Immunol 2002; 2: 725-734.

30. Sun SC. The non-canonical NF-kappaB pathway in immunity and inflammation. Nat Rev Immunol 2017; 17: 545-558.

31. Muslin AJ. MAPK signalling in cardiovascular health and disease: Molecular mechanisms and therapeutic targets. Clin Sci (Lond) 2008; 115: 203-218.

32. Westerterp M, Bochem AE, Yvan-Charvet L, Murphy AJ, Wang $\mathrm{N}$, Tall AR. ATP-binding cassette transporters, atherosclerosis, and inflammation. Circ Res 2014; 114: 157-170.

33. Tang C, Liu Y, Kessler PS, Vaughan AM, Oram JF. The macrophage cholesterol exporter ABCA1 functions as an antiinflammatory receptor. J Biol Chem 2009; 284: 32336-32343.

34. Tang C, Houston BA, Storey C, LeBoeuf RC. Both STAT3 activation and cholesterol efflux contribute to the anti-inflammatory effect of apoA-I/ABCA1 interaction in macrophages. $J$ Lipid Res 2016; 57: 848-857.

35. Yin K, Chen WJ, Zhou ZG, Zhao GJ, Lv YC, Ouyang XP, et al. Apolipoprotein A-I inhibits CD40 proinflammatory signaling via ATP-binding cassette transporter A1-mediated modulation of lipid raft in macrophages. J Atheroscler Thromb 2012; 19: 823 836.

36. Liu D, Ding Z, Wu M, Xu W, Qian M, Du Q, et al. The apolipoprotein A-I mimetic peptide, D-4F, alleviates ox-LDL-induced oxidative stress and promotes endothelial repair through the eNOS/HO-1 pathway. J Mol Cell Cardiol 2017; 105: 77-88.

37. Wu BJ, Ong KL, Shrestha S, Chen K, Tabet F, Barter PJ, et al. Inhibition of arthritis in the Lewis rat by apolipoprotein A-I and reconstituted high-density lipoproteins. Arterioscler Thromb Vasc Biol 2014; 34: 543-551.

\section{Supplementary Files}

\section{Supplementary File 1}

Figure S1. AIBP effects the LPS-stimulated secretion of inflammatory factors in HepG-2 cells.

Please find supplementary file(s);

http://dx.doi.org/10.1253/circj.CJ-17-0877 